OPEN ACCESS

Edited by:

Akio Adachi,

Kansai Medical University, Japan

Reviewed by:

Xiaozhen Liang,

Institut Pasteur of Shanghai (CAS),

China

Laurie T. Krug,

National Cancer Institute,

United States

Tiffany Reese,

University of Texas Southwestern Medical Center, United States

*Correspondence:

Leslie J. Berg

leslie.berg@cuanschutz.edu

Specialty section This article was submitted to

Virology,

a section of the journal

Frontiers in Microbiology

Received: 21 January 2021

Accepted: 15 February 2021

Published: 09 March 2021

Citation:

Riggs JB, Medina EM

Perrenoud LJ, Bonilla DL, Clambey ET, van Dyk LF and Berg LJ (2021) Optimized Detection of Acute MHV68 Infection With a Reporter System Identifies Large Peritoneal Macrophages as a Dominant Target of Primary Infection.

Front. Microbiol. 12:656979. doi: 10.3389/fmicb.2021.656979

\section{Optimized Detection of Acute MHV68 Infection With a Reporter System Identifies Large Peritoneal Macrophages as a Dominant Target of Primary Infection}

\author{
Julianne B. Riggs' ${ }^{1}$ Eva M. Medina', Loni J. Perrenoud' ${ }^{1}$, Diana L. Bonilla², \\ Eric T. Clambey ${ }^{3}$, Linda F. van Dyk ${ }^{1}$ and Leslie J. Berg ${ }^{1 *}$ \\ ${ }^{1}$ Department of Immunology and Microbiology, University of Colorado Anschutz Medical Campus, Aurora, CO, \\ United States, ${ }^{2}$ Cytek Biosciences, Fremont, CA, United States, ${ }^{3}$ Department of Anesthesiology, University of Colorado \\ Anschutz Medical Campus, Aurora, CO, United States
}

Investigating the dynamics of virus-host interactions in vivo remains an important challenge, often limited by the ability to directly identify virally infected cells. Here, we utilize a beta-lactamase activated fluorescent substrate to identify primary targets of murine gammaherpesvirus 68 (MHV68) infection in the peritoneal cavity. By optimizing substrate and detection conditions, we were able to achieve multiparameter characterization of infected cells and the ensuing host response. MHV68 infection leads to a pronounced increase in immune cells, with CD8+ T cells increasing by 3 days, and total infiltrate peaking around 8 days post-infection. MHV68 infection results in near elimination of large peritoneal macrophages (LPMs) by 8 days post-infection, and a concordant increase in small peritoneal macrophages (SPMs) and monocytes. Infection is associated with prolonged changes to myeloid cells, with a distinct population of MHC II ${ }^{\text {high }}$ LPMs emerging by 14 days. Targets of MHV68 infection could be readily detected. Between 1 and 3 days post-infection, MHV68 infects $\sim 5-10 \%$ of peritoneal cells, with $>75 \%$ being LPMs. By 8 days post-infection, the frequency of MHV68 infection is reduced at least 10-fold, with infection primarily in SPMs, with few infected dendritic cells and B cells. Importantly, limiting dilution analysis indicates that at 3 days post-infection, the majority of MHV68-infected cells harbor latent rather than lytic virus at frequencies consistent with those identified based on reporter gene expression. Our findings demonstrate the utility of the beta-lactamase MHV68 reporter system for high throughput single-cell analysis and identify dynamic changes during primary gammaherpesvirus infection.

Keywords: gammaherpesvirus, macrophages, peritoneal cavity, latency, reporter virus, multiparameter flow cytometry 


\section{INTRODUCTION}

The gammaherpesviruses ( $\gamma \mathrm{HVs}$ ) are a highly conserved family of DNA tumor viruses characterized by their capacity to establish lifelong, latent infection in their hosts. The $\gamma \mathrm{HVs}$ include the human viruses, Epstein-Barr virus (EBV), and Kaposi's-associated sarcoma virus (KSHV), multiple primate $\gamma \mathrm{HVs}$ and murine gammaherpesvirus 68 (MHV68, official ICTV nomenclature MuHV-4) (Barton et al., 2011; Dittmer and Damania, 2016; Young et al., 2016). For most individuals, $\gamma \mathrm{HV}$ infection is well-controlled, with no overt deleterious consequences. However, particularly in immunosuppressed individuals, chronic $\gamma \mathrm{HV}$ infection is associated with the development of malignancies, including lymphomas and carcinomas (Cesarman et al., 2019; Farrell, 2019; Shannon-Lowe and Rickinson, 2019).

The $\gamma \mathrm{HV}$ s result in a lifelong infection of their hosts, involving a number of different cell types over time. In vivo, MHV68 is thought to lytically replicate in epithelial cells of mucosal surfaces (e.g., nasopharynx and lung) following mucosal exposure, with long-term latent infection primarily occurring in memory B cells in secondary lymphoid organs such as the spleen (SunilChandra et al., 1992; Frederico et al., 2012; Lawler et al., 2015). Additionally, MHV68 has been reported in various tissues depending on the route of infection [peritoneal cavity (Weck et al., 1999), lymph node, omentum (Gray et al., 2012), bone marrow (Coleman et al., 2010), and gut (Peacock and Bost, 2000; Cho et al., 2019, 2020)], with various types of infection observed in B cell subsets (Flano et al., 2000; Coleman et al., 2010; Rekow et al., 2016; Collins et al., 2020), myeloid cells (macrophages and dendritic cells) (Weck et al., 1999; Flano et al., 2000), endothelial cells (Suarez and van Dyk, 2008), and intestinal epithelial cells (Peacock and Bost, 2000). The vast array of potential viral reservoirs calls for an efficient and robust method to track infection.

In the past, limiting dilution analyses were used to track MHV68 in various tissues (Barton et al., 2011). These methods are highly sensitive, but limited in their ability to identify specific cell types that are targets of infection. A variety of MHV68 reporter viruses have been generated. These reporter viruses are able to be used with single-cell immunophenotyping methods, such as flow cytometry and immunohistochemistry. However, these fluorescent proteins are expressed only in the early stages of infection and can be difficult to detect in autofluorescent cell types (Rosa et al., 2007; Hwang et al., 2008; Collins et al., 2009; Collins and Speck, 2012).

The use of a beta-lactamase tagged virus, MHV68.LANA: Blac, expands upon these capabilities (Nealy et al., 2010). The betalactamase gene is linked to ORF73, a viral gene encoding LANA, the episome-maintenance protein and transcription factor that is expressed throughout the viral lifecycle. It is notable that while the LANA protein is expressed throughout infection, it is generally expressed at a low level, and thus, combination with the enzymatic amplification of beta-lactamase is critical. Further development of this beta-lactamase tagged virus system allowed tracking of cells infected with panels of mutant viruses both in vitro and in vivo, as well as live cell sorting of rare infected cells for downstream analyses of function and multiparametric mass cytometry and transcriptomics (Diebel et al., 2015; Berger et al., 2020).

The peritoneal cavity (PerC) is an important and relevant tissue of immunological function, as well as a source of easily isolated immune cells. Despite many viral infections being delivered intraperitoneally, the immune response to infection in this cavity is not well characterized. The cavity is composed primarily of macrophages and B cells (Ray and Dittel, 2010). Peritoneal B cells are predominantly B-1 cells, "innate" B cells that produce natural antibodies (Baumgarth, 2017). MHV68 has been identified to establish latency in peritoneal macrophages and B-1 cells (Weck et al., 1999; Rekow et al., 2016). Macrophages are of two types: large peritoneal macrophages (LPMs), characterized as tissue resident, self-renewing "clean-up" phagocytes, and small peritoneal macrophages (SPMs), which are more inflammatory, and replaced from circulating monocytes in the blood (Ghosn et al., 2010). LPMs are the dominant population, however it is SPMs that are elicited from thioglycolate stimulation, a common method in obtaining peritoneal macrophages (Ghosn et al., 2010). Lytic infection of primary macrophages has also been established in vitro (Tarakanova et al., 2007; Rosa et al., 2007) but characterization of acute gammaherpesvirus infection of the PerC is limited.

Here, we optimized detection of a reporter virus, MHV68.LANA:ßlac, using full spectrum (i.e., spectral) multiparameter flow cytometry to achieve single cell resolution of virus infection during primary infection in the PerC. We show that MHV68 infection in the PerC leads to dramatic changes in immune cell populations, most notably a transient elimination of LPMs and an increase in SPMs, monocytes, and CD8+ T cells. We use MHV68.LANA: $\beta$ lac to track infection over time, identifying, and immunophenotyping virally infected cells up to 2 weeks post infection. We show that LPMs represent the dominant reservoir of MHV68 during acute infection, and that infection in the PerC at this time is a mixture of both lytic and, predominantly, latent infection. These studies demonstrate a method for expansive immunophenotyping that can be integrated with detection of the MHV68.LANA:ßlac reporter virus, and present new insights in acute gammaherpesvirus infection.

\section{MATERIALS AND METHODS}

\section{Mice}

C57BL/6 mice were purchased from Jax and bred in house (Catalog \#00664). Mice were maintained at the University of Colorado Anschutz Medical Campus in accordance with IACUC protocol and were used for experiments at 6-12 weeks of age. Both male and female mice were used.

\section{Viral Infections}

Wild-type (WT) MHV68 and MHV68.LANA:ßlac viruses were grown and prepared as described previously (Diebel et al., 2015). For in vitro infections, mouse $3 \mathrm{~T} 12$ fibroblasts and ex vivo peritoneal cells were infected overnight, with no removal of 
inoculum, at a multiplicity of infection (MOI) of 1 and 10 plaque forming units (PFU) per cell, respectively. Cells were cultured in DMEM with L-glutamine, $1 \%$ Penicillin/Streptomycin and $10 \%$ FBS. For in vivo infections, mice were inoculated intraperitoneally (i.p.) with $1 \times 10^{6} \mathrm{PFU}$ of virus diluted in sterile PBS.

\section{Harvest of Peritoneal Cells}

Mice were sacrificed via $\mathrm{CO}_{2}$ euthanasia and cervical dislocation. The PerC was exposed and filled with $10 \mathrm{~mL}$ of ice cold PBS containing 3\% FBS using a 27 gage needle. After removal of the needle, the mouse was then agitated to encourage release of immune cells from the peritoneal wall, followed by fluid and cell extraction using an 18 gage needle. If necessary, cells were subjected to red blood cell lysis with ACK lysis buffer (Gibco Catalog \#A1049201), washed and counted using the Beckman Coulter Vi-CELL BLU.

\section{Flow Cytometric Analysis of Peritoneal Cells}

Following harvest and preparation, peritoneal cells were subjected to flow cytometric analysis. First, cells were assessed for viability using a $30 \mathrm{~min}$ RT incubation with Zombie NIR (BioLegend) diluted in PBS. CCF2-AM substrate

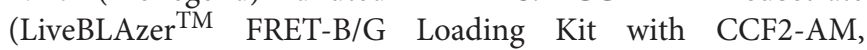
Thermo Fisher Scientific) (Zlokarnik et al., 1998) was freshly diluted prior to substrate loading for 30-60 min RT in PBS. Cells were subjected to Fc receptor blocking using Fc Shield (Tonbo Biosciences) for $10 \mathrm{~min}$, followed by antibody staining for $25 \mathrm{~min}$. The following antibodies, with antibody clones designated in parenthesis, were used: CD19(1D3)PECy7, CD11b(M1/70)-APC (eBiosciences/Thermo Fisher Scientific), CD11c(HL3)-BUV737, CD4(GK1.5)-BUV395, CD8a(53-6.7)-PerCPCy5.5 (BD), CD3e(145-2C11)-PECy5, F4/80(BM8)-BV785, MHC II(M5/14.15.2) PerCP, Gr1(RB68C5)-AlexaFluor700, Gal3(M3/38)-AlexaFluor647 (BioLegend). All staining reactions were done at RT in the dark in PBS for antibody stains and PBS containing 1\% FBS for Fc receptor blockade. Samples were acquired on a Cytek Aurora Spectral Analyzer with five lasers (UV, violet, blue, yellow-green, and red) using SpectroFlo software. Gating for specific cell populations, as well as for CCF2, was determined using control samples for each run. Differences in gating between time points reflect variations in experimental and spectral analyzer conditions on different days. Detailed information concerning unmixing of CCF2 and effects of fixation and permeabilization of CCF2 on fluorescence can be provided upon request.

\section{MHV68 Reactivation Assay}

C57BL/6 mouse embryonic fibroblasts (MEFs) were cultured in DMEM with L-glutamine, 20\% FBS, 1\% Penicillin/Streptomycin and $1 \times$ Amphotericin B at 5,000 cells per well in 96-well flat bottom tissue culture-treated plates. Peritoneal cells (PerCs) were harvested from mice inoculated with PBS alone, MHV68 or MHV68.LANA:ßlac at 3 days post infection (dpi). PerCs were washed, counted, and diluted to the indicated concentrations.
In parallel, cells were subjected to mechanical disruption and plated in a comparable series of dilutions prior to plating on MEF monolayers (Niemeyer et al., 2018). Co-cultures were incubated at $37^{\circ} \mathrm{C}$ for 21 days prior to assessment of cytopathic effect (CPE). A dashed line at $63.2 \%$ was used to determine the frequency of virus-positive cells, defined by Poisson distribution. Analysis was performed via non-linear regression analysis in GraphPad Prism, with calculation of $95 \%$ confidence intervals. Data represents three experiments with two mice per condition per experiment.

\section{Data Analysis}

Analysis of flow cytometry data was performed using Cytek SpectroFlo version 2.1 and DeNovo FCS Express version 7. Fluorophore signatures with partial overlap of emission spectra were resolved by unmixing using SpectroFlo software without compensation. Statistical analysis was performed using student's $t$-test in GraphPad Prism version 8.

\section{RESULTS}

\section{Optimization of MHV68.LANA: $\beta$ lac Detection Analyzing CCF2-AM Substrate Cleavage by Spectral Flow Cytometry}

We sought to harness the powerful technology of multiparametric flow cytometry with the beta lactamase reporter virus to track virally infected cells in vitro and in vivo. Previous use of a beta-lactamase expressing MHV68 virus detected virally infected cells utilizing conventional, band-pass flow cytometry (Coleman et al., 2010; Nealy et al., 2010; Diebel et al., 2015; Niemeyer et al., 2018). Due to the broad emission spectrum of the beta-lactamase substrate, this approach was limited in its utility for simultaneous measurement of multiple fluorescent markers. In this study, we utilized the power of full spectrum (herein referred to as spectral) flow cytometry. This technique characterizes fluorophores by their entire spectra, rather than peak emission, allowing for the detection of fluorophores relatively close in peak emission and thus vastly expanding the number of fluorophores that can be simultaneously analyzed. Full spectrum flow cytometry also allows for the detection of fluorescent reporters and proteins whose spectra are not well characterized or are unknown (Nolan and Condello, 2013; Nolan et al., 2013).

Studies with the MHV68.LANA:ßlac reporter virus rely on detection of cleavage of a beta-lactamase substrate, such as CCF2AM (Zlokarnik et al., 1998; Knapp et al., 2003), an esterified beta-lactamase substrate that readily crosses the cell membrane (Figure 1). Once inside the cell, endogenous cytoplasmic esterases cleave ester groups, resulting in the lipophobic molecule CCF2. CCF2 consists of a coumarin (identified as " $\mathrm{A}$ " in Figure 1A) linked to a fluorescein (identified as "B," Figure 1A) via a cephalosporin group. The coumarin group absorbs light at a maximum wavelength of $408 \mathrm{~nm}$ and, via Fluorescence Resonance Energy Transfer (FRET), transfers the energy to the fluorescein group, which emits at a maximum wavelength of $520 \mathrm{~nm}$. In the uncleaved state, CCF2 (Figure 1A, left) 


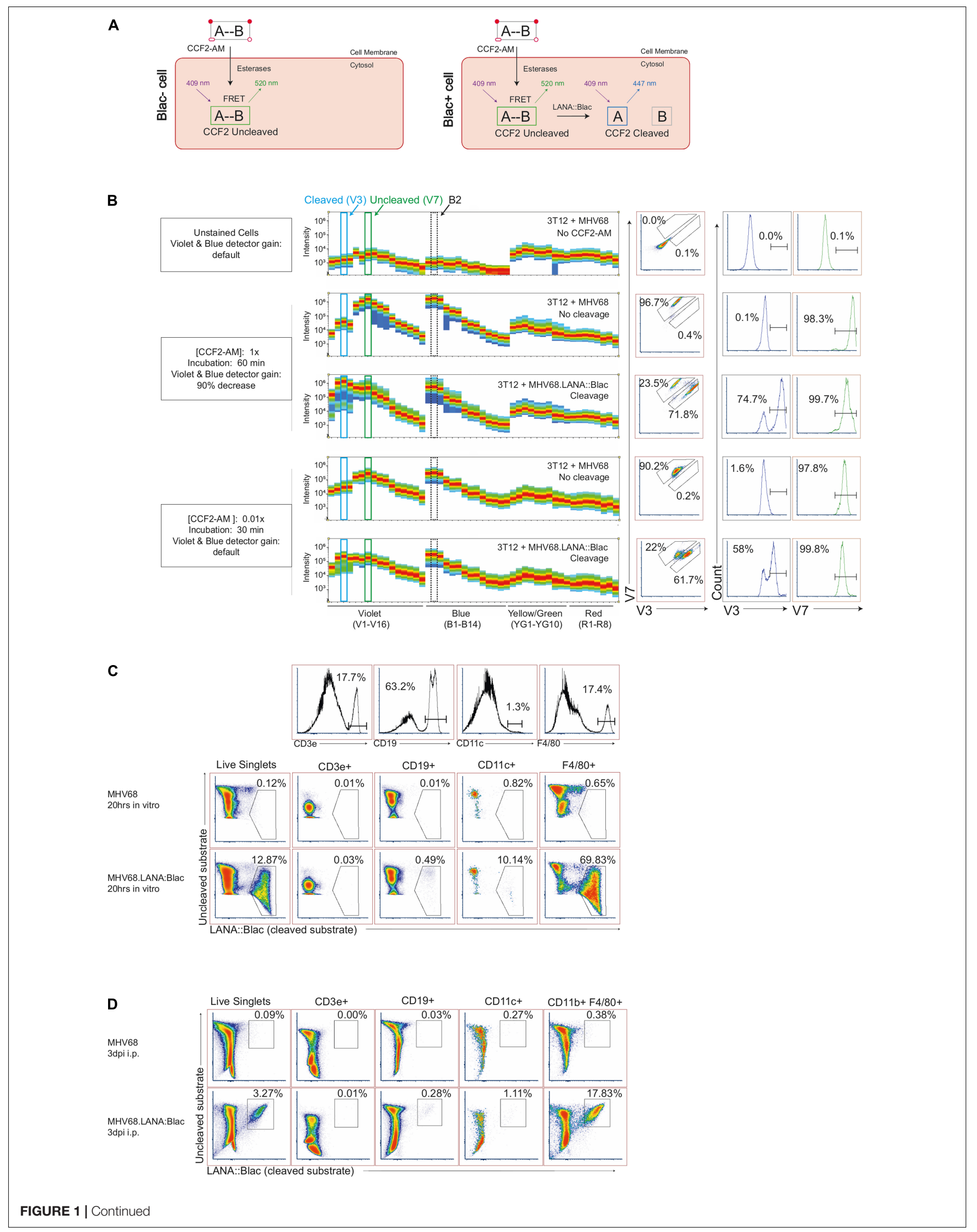


FIGURE 1 | Optimization of MHV68.LANA:ßlac detection and CCF2-AM cleavage by full spectrum flow cytometry. (A) Graphic depicting CCF2-AM entrance, processing and fluorescence in cells in the absence (left) or presence (right) of mLANA:ßlac. "A" represents the coumarin component of CCF2, while "B" represents fluorescein. (B) CCF2-AM titration and detection in 3T12 mouse fibroblasts, infected with either MHV68 (i.e., no CCF2-AM cleavage) or MHV68.LANA: $\beta$ lac (i.e., CCF2-AM cleavage) and incubated with the indicated concentration of CCF2-AM at $20 \mathrm{~h}$ post-infection. Shown are full emission spectra from stained cells. Each row depicts fluorescence intensity under different experimental conditions (MHV68 or MHV68.LANA:Blac infection), varying CCF2-AM concentration and incubation time, and gain for Violet and Blue detectors of a Cytek Aurora spectral analyzer. Top row: unstained fibroblasts. Middle rows: fibroblasts subjected to manufacturer's recommended conditions for CCF2-AM staining. Bottom rows: fibroblasts subjected to optimized, titrated CCF2-AM staining. Each row depicts from left to right: fluorescent spectral intensity across 48 detectors, with fluorescence emission in the V3 and V7 detectors highlighted by dot plot and histogram. CCF2-AM associated fluorescence for the uncleaved substrate is detected in V7, with cleaved substrate detected in V3. (C) Dot plots showing optimized staining conditions in infected mouse peritoneal cells (PerCs) in vitro. PerCs were extracted and infected overnight in vitro with MHV68 or MHV68.LANA:Blac. Twenty hours later, cells were stained with CCF2-AM as well for viability and surface markers. Histograms are shown to demonstrate how populations were defined. (D) Dot plots showing optimized staining conditions on infected PerCs in vivo. Mice were infected i.p. with 1 million PFUs of MHV68 or MHV68.LANA:Blac. Three days after infection, mice were sacrificed and PerCs were harvested and stained with CCF2-AM as well as for viability and surface markers. Populations shown were gated on live singlets. Data representative of three experiments, with 2-3 mice per condition per experiment.

exhibits peak emission from the fluorescein group. In the presence of beta-lactamase enzymatic activity (Figure 1A, right), the cephalosporin group is cleaved to separate the coumarin and fluorescein groups, disrupting FRET and altering the resulting fluorescence to the coumarin group, which emits at a maximum wavelength of $447 \mathrm{~nm}$. Thus, the presence of beta-lactamase ( $\beta$ lac) enzyme, such as the LANA:ßlac fusion protein, can be detected by the unique fluorescence profile of the cleaved CCF2 substrate.

To examine the MHV68.LANA:ßlac reporter system by spectral flow cytometry, we infected mouse 3T12 fibroblasts with either WT MHV68, which lacks $\beta$ lac expression, or MHV68.LANA:Blac for $20 \mathrm{~h}$ at an MOI of $1 \mathrm{PFU} /$ cell, and incubated cells with a final concentration of $10 \mu \mathrm{M}$ CCF2AM substrate according to manufacturer's recommendations. When cells were analyzed for fluorescence on a Cytek Aurora spectral analyzer, both MHV68 and MHV68.LANA:ßlac infected samples were characterized by an extremely bright fluorescent signal (Figure 1B), consistent with what had been observed in previous reports with conventional band pass flow cytometry (ETC, personal communication). Maximal fluorescence of the uncleaved CCF2 substrate, in MHV68infected cultures, was detected in the V7 and B2 fluorescent detectors (Figure 1B, second row), consistent with fluorescein emission. In contrast, MHV68.LANA:Blac-infected cultures demonstrated an additional fluorescence maximum in the V3 fluorescent detector, consistent with LANA:ßlac-dependent substrate cleavage. We propose that retention of fluorescence in the $\mathrm{V} 7$ and $\mathrm{B} 2$ detectors likely results from incomplete cleavage of CCF2 in LANA: $\beta l a c+$ cells, a phenomenon also observed in other reports using this system (Zlokarnik et al., 1998; Nealy et al., 2010; Diebel et al., 2015). Despite the ability to detect a unique fluorescent signature in MHV68.LANA:ßlac-infected cells, CCF2-associated fluorescence in these conditions was so great that the original Cytek assay gain settings for the violet and blue detectors had to be decreased by more than $90 \%$. This reduction severely compromised the potential to analyze additional fluorophores excited primarily by these lasers.

To address this limitation, we analyzed the impact of CCF2AM substrate concentration and incubation time on fluorescent emission. Notably, use of $0.1 \mu \mathrm{M}$ CCF2-AM, a 100-fold dilution in substrate, produced a signal that was readily detectable and on-scale yet did not require altering laser voltages on the cytometer (Figure 1B, bottom two rows). We further determined that incubation with the CCF2-AM substrate for half the recommended time $(30 \mathrm{~min}$ instead of $60 \mathrm{~min}$ ) still produced a robust signal (Figure 1B). These optimized substrate conditions afforded the opportunity to discriminate between uncleaved substrate, resulting in maximal fluorescence in the V7 and B2 detectors, and cleaved CCF2 substrate, uniquely fluorescing in the V3 detector while retaining fluorescence in the V7 and B2 detectors. Significantly, these settings did not require compromised gains for either the blue or violet laser, allowing detection of other fluorophores. In our optimization studies, we also determine the best method for unmixing cleaved and uncleaved substrate, as well as the effect of fixation and permeabilization on the CCF2 fluorescent signal. Detailed information on these methods will be provided by the authors upon request.

To determine if our optimized CCF2-AM staining conditions worked in primary cells, we infected mouse peritoneal cells (PerCs) directly ex vivo. The PerC is rich in macrophages and B cells, two cell types known to be latently infected following MHV68 infection (Weck et al., 1999; Rekow et al., 2016). PerCs were isolated and incubated overnight in vitro with either WT MHV68 or MHV68.LANA:ßlac (MOI $=10 \mathrm{PFUs} / \mathrm{cell}$ ), followed by use of optimized CCF2-AM incubation conditions. WT MHV68 infected cultures had minimal fluorescence in the CCF2 cleaved substrate detection channel (Figure 1C, top row). In contrast, $\sim 12 \%$ of MHV68.LANA:ßlac infected PerCs demonstrated CCF2 cleaved substrate-dependent fluorescence (Figure 1C, bottom row). Furthermore, these conditions allowed us to use additional fluorophores excited by the violet and blue lasers, a result unachievable using the manufacturer's recommended staining conditions. The use of multiple antibodies to detect a panel of cell surface markers allowed us greater resolution to immunophenotype virus-positive cells. MHV68.LANA: $\beta$ lac was detected in a high frequency of F4/80+ macrophages, with a lower frequency of cleaved substrate detected in $\mathrm{CD} 11 \mathrm{c}+$ dendritic cells and $\mathrm{CD} 19+\mathrm{B}$ cells, and no detectable fluorescence in CD3+ T cells (Figure 1C). Next, we tested our ability to detect MHV68.LANA:Blac infection in PerCs harvested from mice that were subjected to intraperitoneal infection and harvested at 3 days post-infection (dpi). Again, a 
A

PerC

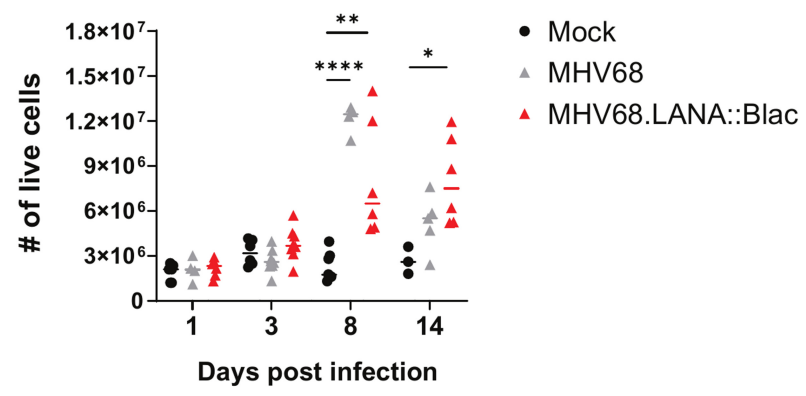

B

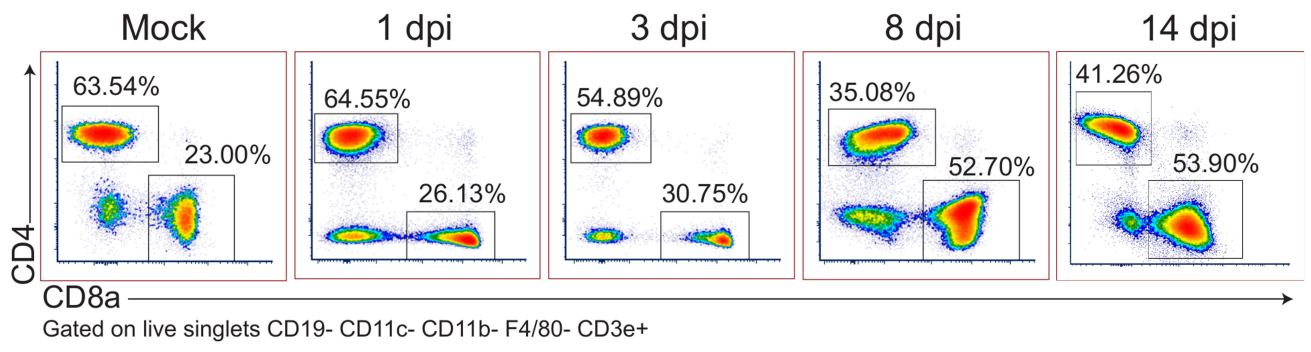

C
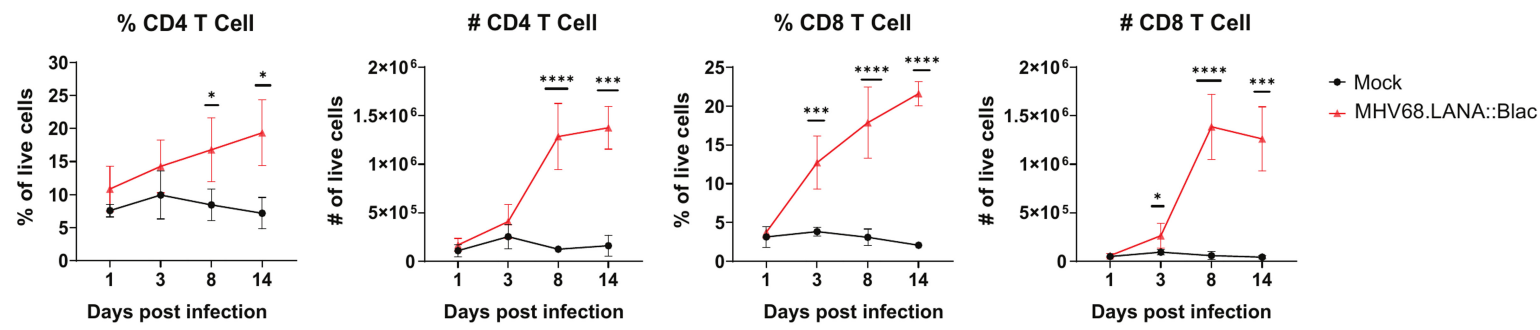

FIGURE 2 | Intraperitoneal MHV68.LANA:Blac infection induces T cell accumulation in the peritoneal cavity. Experimental conditions were performed as outlined in Figure 1D. Mice were harvested at 1, 3, 8, and $14 \mathrm{dpi}$. (A) Viable cell counts of PerCs as a function of time and infection cohort. Each symbol is representative of one mouse and bars represent the mean value of samples. Asterisks denote significance between Mock and MHV68 (8 dpi), Mock and MHV68.LANA:Blac ( 8 dpi), and Mock and MHV68.LANA:ßlac (14 dpi). (B) Flow cytometry dot plots showing percent of CD4- and CD8a-positive PECs at various time points post infection with MHV68.LANA:Blac. Samples are gated on live, singlets CD19- CD11c- CD11b- F4/80- CD3e+ cells. (C) Quantification of flow cytometry data showing mean percent and number of CD4 and CD8a-positive PECs are various time points post infection. Statistical analysis was performed using student's t test in GraphPad Prism $\left({ }^{*} p<0.05,{ }^{* *} p<0.01,{ }^{* * *} p<0.001,{ }^{* * * *} p<0.0001\right)$. Data representative of three experiments with $2-3$ mice per condition per experiment.

robust cleaved CCF2 signal was observed, particularly among F4/80+ macrophages (Figure 1D).

With optimization established, we sought to apply this to analyze primary MHV68 infection using MHV68.LANA:ßlac infection and spectral flow cytometry.

\section{MHV68 Results in Dramatic Changes to the Immune Landscape of the Peritoneal Cavity}

The viral lifecycle of $\gamma \mathrm{HVs}$ is dynamic, with significant changes in viral load and cell and tissue tropism over time. To identify the consequence of acute MHV68 infection on cell types and targets of virus infection, mice were infected intraperitoneally (i.p.) with either WT MHV68 or MHV68.LANA:Blac, and analyzed over 14 days of infection. Though i.p. infection is likely not the natural route of infection for this virus, i.p. infections are a common strategy for virus delivery, including MHV68 (Barton et al., 2011). Additionally, MHV68 has a known tropism for peritoneal cells, and i.p. infection establishes robust and temporally synchronized infection within this tissue, providing an opportunity to investigate detailed kinetics of viral infection.

Cell counts revealed that i.p. MHV68 infection triggers a robust inflammatory response in the PerC, peaking at 8 dpi (Figure 2A). This is accompanied by appreciable splenomegaly (Supplementary Figure 1). Leukocyte cell counts began to subside after $8 \mathrm{dpi}$, but remain elevated compared to mock-infected mice. Despite host cell counts in response to MHV68.LANA:Blac infection appearing more variable than those following MHV68 infection, no significant differences were observed between these two experimental groups. MHV68 infection induced a prominent increase in $\mathrm{T}$ cell frequency 
and number, dominated by CD8+ $\mathrm{T}$ cells (Figures 2B,C). Notably, CD8 $\mathrm{T}$ cells increased by $3 \mathrm{dpi}$, indicating rapid engagement of $\mathrm{CD} 8 \mathrm{~T}$ cells. Immune cell populations were defined as according to the gating strategy detailed in Supplementary Figure 2.

Murine gammaherpesvirus 68 infection was also associated with pronounced changes in myeloid subsets. In naïve mice, there are two dominant macrophage populations, LPMs, defined as $\mathrm{F} 4 / 80^{\mathrm{High}} \mathrm{CD} 1 \mathrm{~b}^{\mathrm{High}} \mathrm{MHC} \mathrm{II}^{\mathrm{Low}}$, and $\mathrm{SPM}$, defined as F4/80 ${ }^{\text {Mid }} \mathrm{CD}_{11} \mathrm{~b}^{\text {Mid }}$ MHC II ${ }^{\text {High }}$ (Ghosn et al., 2010). MHV68 infection reduced the frequency of LPMs relative to mockinfected mice, from day 1 through day 14 post-infection, with no LPMs detected at $8 \mathrm{dpi}$ and an apparent reappearance by day 14 (Figures $3 \mathbf{A}, \mathbf{B}$ ). This dramatic loss of LPMs was accompanied by a pronounced increase in the frequency and number of $\mathrm{F} 4 / 80^{\mathrm{Mid}} \mathrm{CD} 11 \mathrm{~b}^{\mathrm{Mid}}$ cells, consistent with an SPM or monocyte phenotype, between days 3 and 14 post-infection (Figures 3A,B). The phenotype of $\mathrm{F} 4 / 80^{\mathrm{Mid}} \mathrm{CD} 11 \mathrm{~b}^{\mathrm{Mid}}$ cells changed over time. In mock-infected mice, $\mathrm{F} 4 / 80^{\mathrm{Mid}} \mathrm{CD} 11 \mathrm{~b}^{\mathrm{Mid}}$ cells were predominantly a Gr1 ${ }^{\mathrm{Low}} \mathrm{MHC} \mathrm{II}^{\mathrm{High}}$ phenotype consistent with SPMs (Ghosn et al., 2010). Following MHV68 infection, however, $\mathrm{F} 4 / 80^{\mathrm{Mid}} \mathrm{CD} 11 \mathrm{~b}^{\mathrm{Mid}}$ cells demonstrated a transient increase in the frequency of $\mathrm{Gr}^{\mathrm{High}} \mathrm{MHC} \mathrm{II}^{\mathrm{Low}}$ cells, consistent with monocyte infiltration into the PerC (Figure 3C). By day 8 and 14 post-infection, $\mathrm{F} 4 / 80^{\mathrm{Mid}} \mathrm{CD} 11 \mathrm{~b}^{\mathrm{Mid}}$ cells were primarily $\mathrm{Gr} 1^{\mathrm{Low}} \mathrm{MHC} \mathrm{II}^{\mathrm{High}}$ cells, consistent with SPMs (Figure 3C).

The disappearance and reappearance of the LPM population by 14 dpi suggested self-renewal and/or replenishment. Despite prior reports detailing LPMs as self-renewing (Cassado Ados et al., 2015), the complete absence of LPMs at 8 dpi suggests replenishment from another population. Monocytes from the circulation are a likely candidate, as they have been shown to enter the PerC and acquire LPM characteristics (Bain et al., 2016). Notably, as infection progressed, SPM/monocyte and LPM populations became less distinct based on CD11b and F4/80 expression (e.g., see $14 \mathrm{dpi}$, Figure 3A). SPM and LPM protein expression was further altered by infection. In uninfected mice, SPMs have been characterized as MHC $\mathrm{II}^{\text {High }} \mathrm{CD} 86^{\mathrm{Low}}$, in contrast to LPMs which are MHC $\mathrm{II}^{\mathrm{Low}}$ $\mathrm{CD}^{\text {High }}$ (Ghosn et al., 2010); we also find these distinct phenotypes in mock-infected mice (Figure 3D). In contrast, by 14 and $21 \mathrm{dpi}, \mathrm{F} 4 / 80^{\mathrm{Mid}} \mathrm{CD} 11 \mathrm{~b}^{\mathrm{Mid}} \mathrm{SPM} /$ monocytes upregulated CD86 expression compared to mock-infected conditions (Figure 3D). Conversely, F4/80 $0^{\text {High }} \mathrm{CD} 11 \mathrm{~b}^{\mathrm{High}}$ LPMs increased their expression of MHC II, with LPMs demonstrating an MHC $\mathrm{II}^{\mathrm{High}}$ phenotype by day 14 and 21 dpi (Figure 3D). These phenotypic changes resulted in SPM/monocyte and LPM populations both expressing an MHC $\mathrm{II}^{\mathrm{High}} \mathrm{CD} 86^{\mathrm{High}}$ phenotype by $14 \mathrm{dpi}$, blurring the phenotypic distinction between these cell subsets. In addition to phenotypic changes in macrophage subsets in the PerC, MHV68 infected mice further demonstrated an increase in the frequency of neutrophils in the PerC by $14 \mathrm{dpi}$ (Figure 3E). These data demonstrate multiple time-dependent changes in leukocyte composition and phenotype following primary MHV68 infection.

\section{MHV68.LANA: $\beta$ lac+ Is detectable in macrophages, dendritic cells, and B cells in the peritoneal cavity}

We next analyzed the cellular distribution of MHV68.LANA:Blac infection in the PerC over time. The frequency of LANA:ßlac+ cells was relatively constant between 1 and $3 \mathrm{dpi}$, with at least $5 \%$ of total peritoneal cells demonstrating CCF2-AM substrate cleavage, a single-cell marker for LANA:Blac expression (Figure 4). The frequency of LANA:Blac+ cells decreased significantly $(\sim 10$-fold) by $8 \mathrm{dpi}$, with a further decrease in the frequency of LANA:ßlac+ PerCs by 14 dpi (Figure 4). Despite the decreased frequency of LANA:ßlac+ cells over time, these cells were readily detected above the limit of detection defined by coincident analysis of mock and WT MHV68 infected samples (Figure 4).

We next immunophenotyped PerCs from infected mice using a panel of 12 cell surface markers. As predicted from our initial in vivo experiments (Figure 1D), we detected the virus in multiple cell types, including macrophages, dendritic cells, and $\mathrm{B}$ cells, at varying frequencies (Figure 5). Of these, the majority $(>75 \%)$ of LANA:ßlac + cells were identified within LPMs early after infection ( 1 and $3 \mathrm{dpi}$ ) (Figure 5). By $8 \mathrm{dpi}$, however, F4/80 ${ }^{\text {Mid }} \mathrm{CD} 11 \mathrm{~b}^{\text {Mid }}$ SPMs were the major LANA:Blac+ population (Figure 5), a change in tropism coincident with a nearly complete disappearance of LPMs from the PerC (as shown in Figure 3). B cells and dendritic cells accounted for less than 5\% of LANA:Blac+ cells through 14 dpi (Figure 5). Despite an increase in number over time of infection (Figure 3), neutrophils were not identified as a target of early peritoneal infection (data not shown). Thus, we conclude that macrophages are the major target of MHV68 infection in the PerC during the first 2 weeks of infection.

In parallel, we assessed the percentage of each cell population that was virally infected. This analysis identified a hierarchy of infection 1 day post infection, with $\sim 25 \%$ of LPMs, $7 \%$ of dendritic cells, $5 \%$ of SPM/monocytes and $0.2 \%$ of B cells which were LANA: $\beta l a c+$ (Figure 6). LPMs remained a predominant target of infection at $3 \mathrm{dpi}$, with $>40 \%$ of LPMs characterized by LANA:ßlac expression, in contrast to SPM/monocytes, dendritic cells and B cells which contained $<3 \%$ LANA:ßlac+ cells out to 14 dpi (Figure 6). LANA:Blac expression was not detected in T cells at any time analyzed. These data identify multiple targets of primary MHV68 infection in the PerC.

\section{MHV68 Infection Is Associated With Altered Macrophage Expression of MHC II and CD86 in Infected and Uninfected Cells}

Given the impact of MHV68 infection on myeloid cells, and the partial infection of LPMs and SPMs, we next analyzed how MHV68 infection affected macrophage phenotype in a cellintrinsic and cell-extrinsic manner. To do this, we analyzed MHC II and CD86 expression, two proteins differentially expressed in LPMs and SPMs at baseline (Ghosn et al., 2010), comparing LPMs and SPMs in mock-infected mice with LANA:Blac+ 
A

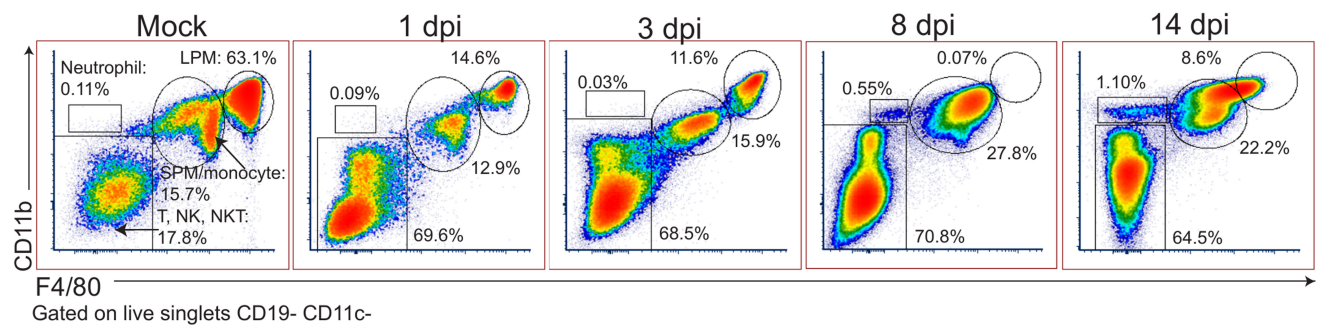

B

$\%$ LPMs

\# LPMs

$\%$ SPM/Monocyte
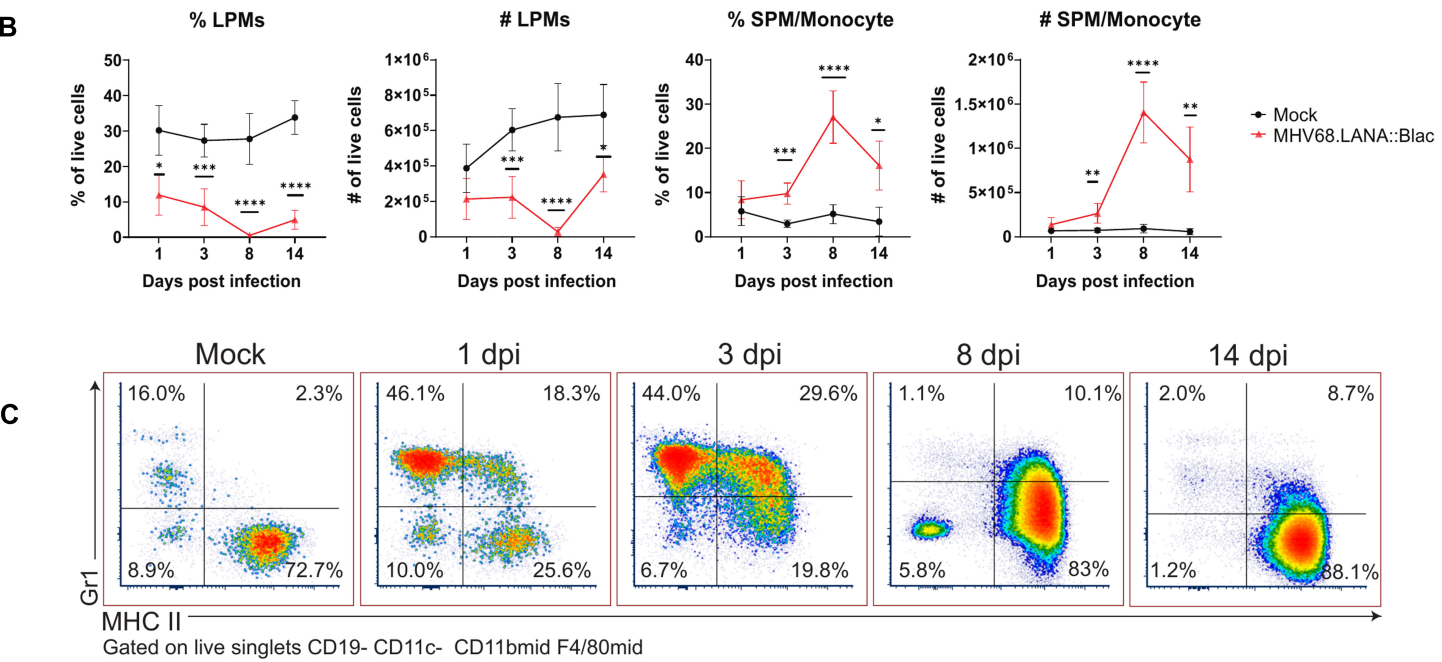

D
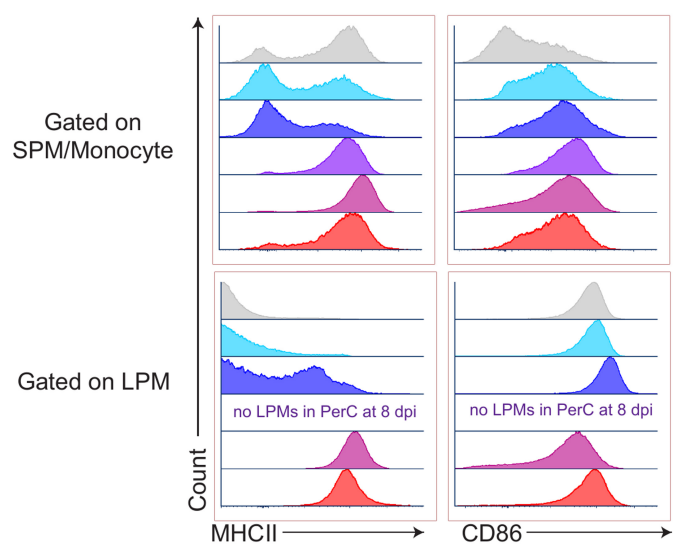

E
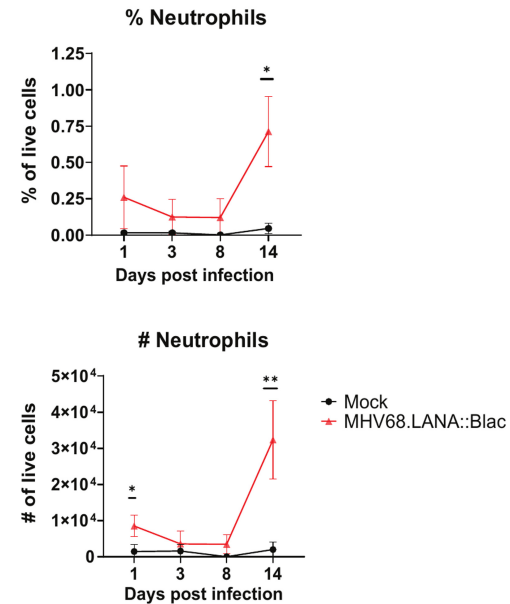

FIGURE 3 | Alterations in peritoneal myeloid subsets after MHV68.LANA:ßlac infection. Flow cytometric analysis of myeloid cells and neutrophils in the peritoneal cavity after MHV68.LANA:Blac infection. (A) Flow cytometric analysis of PerCs, showing expression of CD11b and F4/80 on live, singlet, CD19- CD11C- events at the indicated times post infection. Gates identify distinct cell subsets, including large peritoneal macrophage (LPM) and small peritoneal macrophages (SPM).

(B) Quantification of flow cytometry data shown in (A) showing mean percent and number of live singlets that are LPMs and SPM/monocytes at various times post infection. (C) Dot plots showing expression of Gr-1 and MHC II on CD19- CD11C- CD11bmid F4/80mid PerCs over time. (D) Histograms comparing expression of MHC II (left) and CD86 (right) in SPMs/monocytes (top) and LPMs (bottom), as a function of time (colored according to legend). Day 8 samples did not have any detectable LPMs (as indicated). (E) Quantification of flow cytometry data shown in (A), demonstrating mean percent and number of neutrophils at time points post infection. Statistical analysis was performed using student's $t$-test in GraphPad Prism $\left({ }^{*} p<0.05,{ }^{* *} p<0.01,{ }^{* * *} p<0.001,{ }^{* * * *} p<0.0001\right)$. Data representative of three experiments with 2-3 mice per condition per experiment.

(virus-infected) and LANA:ßlac- (uninfected) cell subsets from infected mice. Timepoints of 1,3 , and 8 dpi were examined; the 14 dpi time point was excluded due to the very low frequency of
LANA:ßlac + events observed in these samples. As anticipated, mock-infected mice contained LPMs with a predominant MHC $\mathrm{II}^{\mathrm{Low}} \mathrm{CD} 86^{\text {High }}$ phenotype (Figure 7, "Mock LPM"). After 1 dpi, 


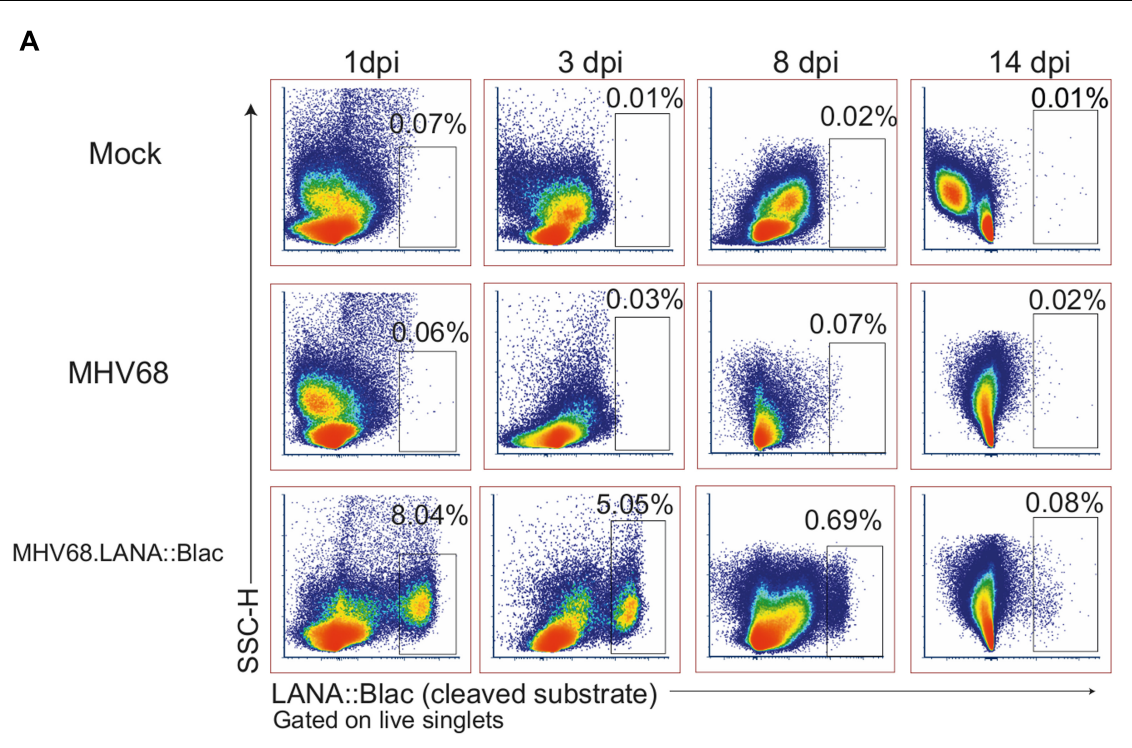

B
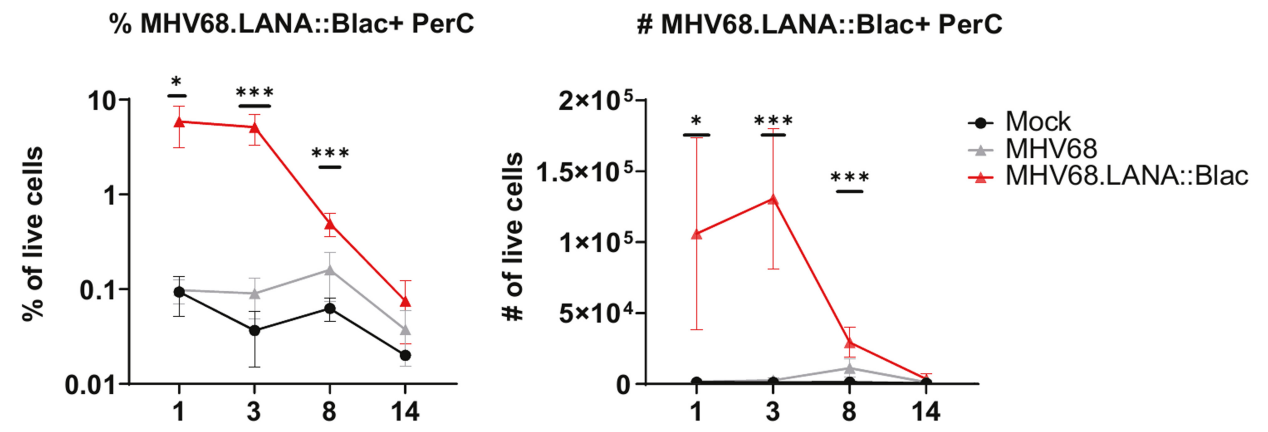

FIGURE 4 | MHV68.LANA: $\beta$ lac is detectable during acute infection in the peritoneal cavity. Flow cytometric analysis of MHV68.LANA: 3 lac infection in the peritoneal cavity of MHV68 infected mice. (A) Dot plots showing the frequency of PerCs positive for cleaved CCF2 fluorescence throughout infection, comparing peritoneal cells isolated from mock, MHV68 or MHV68.LANA: $\beta$ lac infected mice. Populations were gated on live singlets. (B) Quantification of the frequency and number of LANA: $\beta$ lac+ PerCs defined based on positive cleaved CCF2 fluorescence. Statistical analysis was performed using student's $t$ test in GraphPad Prism $\left({ }^{*} p<0.05\right.$, $* * * p<0.001)$. Data representative of three experiments with 2-3 mice per condition per experiment.

$>94 \%$ of LANA:ßlac+ and LANA:ßlac- LPMs were MHC II ${ }^{\text {Low }}$ CD86 ${ }^{\text {High }}$, comparable to mock-infected LPMs (Figure 7, left panel). By 3 dpi, however, MHV68 infected mice demonstrated an increased frequency of MHC $\mathrm{II}^{\mathrm{High}} \mathrm{LPMs}$ compared to mock-infected mice. MHC II ${ }^{\text {High }} \mathrm{CD}^{\text {High }}{ }^{\text {LPMs }}$ were most prominent among virally infected (LANA:ßlac+) LPMs $(49.2 \%$ of events), with an intermediate frequency (23.6\%) in LANA:ßlacLPMs (Figure 7, middle panel). CD86 expression remained high in LPMs in all conditions (Figure 7, bottom panel). These data demonstrate that MHV68 infection is associated with an increased frequency of MHC II ${ }^{\text {High }}$ LPMs, a process that occurs in both virus-infected and -uninfected cells during acute infection.

In parallel, we analyzed the impact of MHV68 infection on the phenotype of $\mathrm{F} 4 / 80^{\mathrm{Mid}} \mathrm{CD} 11 \mathrm{~b}^{\mathrm{Mid}}$ cells, a phenotype containing a mixed population of SPM and monocytes (referred to collectively as SPM/monocytes). Mock-infected $\mathrm{SPM} /$ monocytes were primarily characterized by an MHC II ${ }^{\mathrm{High}}$
CD86 ${ }^{\text {Low }}$ phenotype (Figure 7). After overnight infection and through 3 dpi, LANA: $\beta$ lac + SPM/monocytes had a relatively comparable frequency of $\mathrm{MHC} \mathrm{II}^{\mathrm{High}}$ events comparable to mock-infected mice. In contrast, LANA:ßlac- SPM/monocytes at these early times had a reduced frequency of $\mathrm{MHC} \mathrm{II}^{\mathrm{High}}$ events compared to mock-infected cells (Figure 7). By $8 \mathrm{dpi}$, however, both LANA:ßlac+ and LANA:ßlac- SPM/monocytes had an increased frequency of MHC $\mathrm{II}^{\text {High }}$ events relative to mock-infected mice. While CD86 expression was comparable in all measured SPM/monocyte populations after overnight infection, LANA: $\beta$ lac + demonstrated a transient upregulation at 3 dpi relative to mock-infected and LANA:ßlac- cells, with both LANA: $\beta l a c+$ and LANA: $\beta l a c-$ cells characterized by a modest upregulation of CD86 by 8 dpi (Figure 7). In total, these data identify that MHV68 infection is associated with enhanced MHC II and CD86 expression in virally infected and -uninfected macrophage subsets in the PerC. 
A

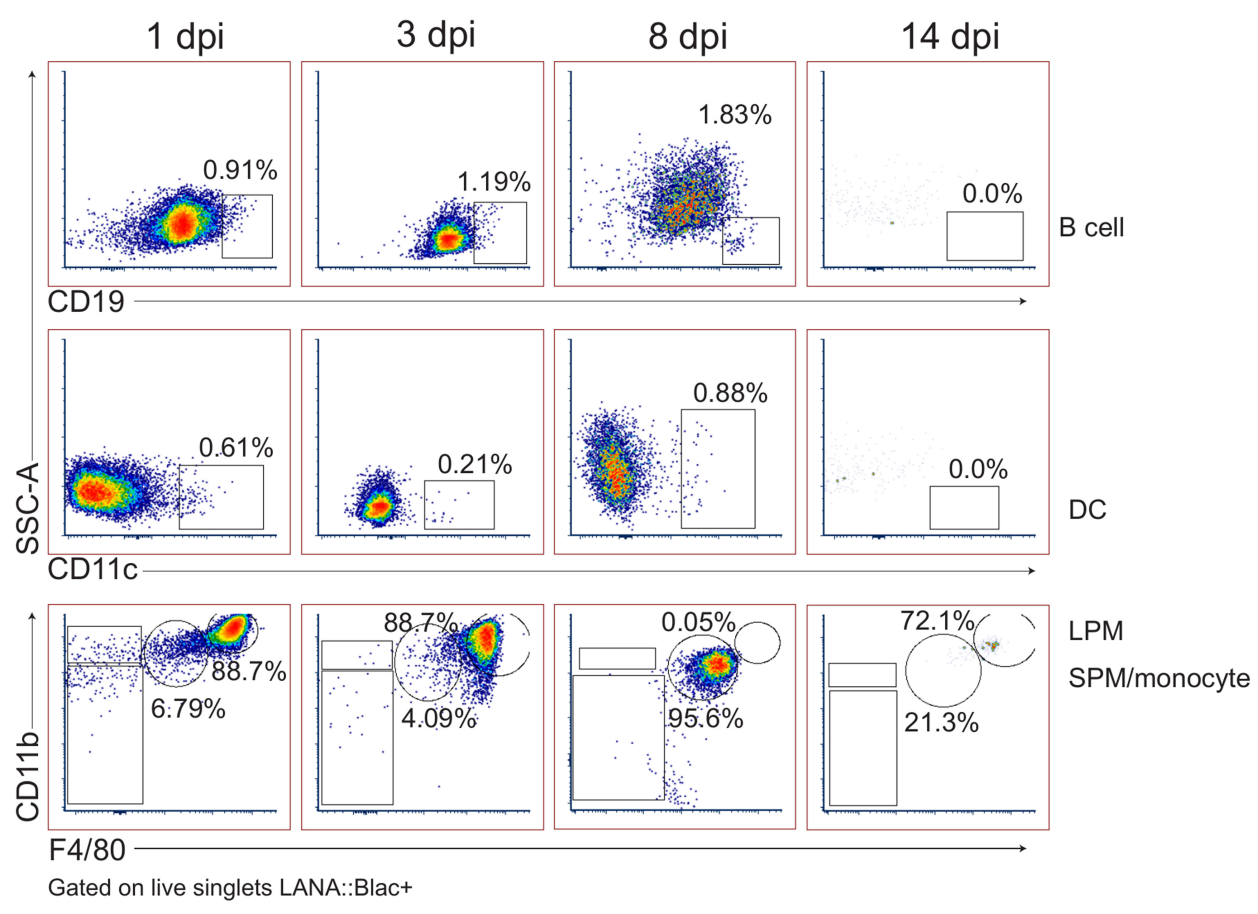

B
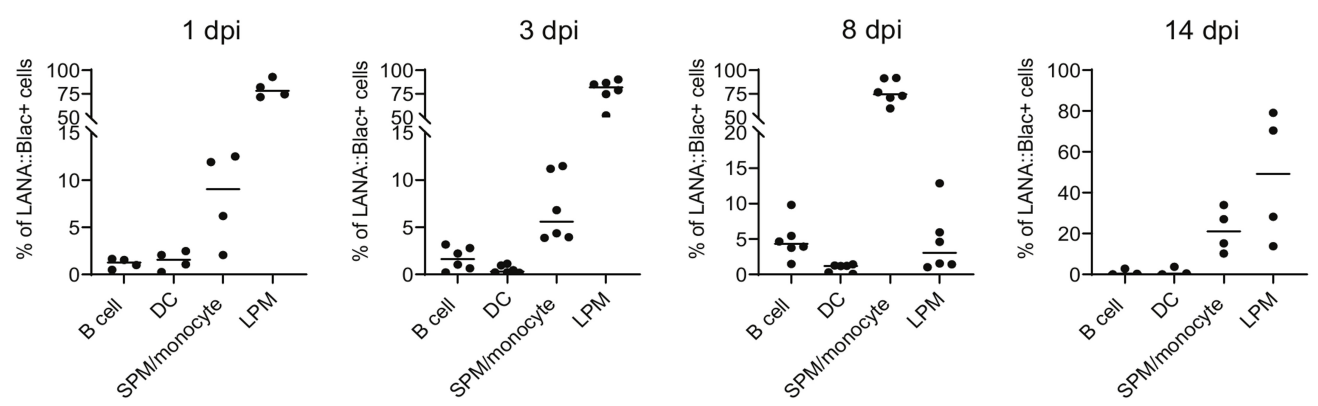

FIGURE 5 | Predominant detection of MHV68.LANA:Blac infection in macrophages in the peritoneal cavity. Flow cytometric analysis of MHV68.LANA:Blac infection within different leukocyte subsets in the peritoneal cavity of MHV68. LANA: $\beta$ lac infected mice. (A) Dot plots quantifying the frequency of LANA: $\beta$ lac+ events (as defined in Figure 4) that are CD19+ B cells (top row), CD11C+ dendritic cells (middle row), or CD11b High $^{\text {F } 4 / 80^{\text {High }} \text { LPMs or CD11b }}$ Mid $_{\text {, F4/80 }}$ Mid SPM/monocytes (bottom row). (B) Quantitation of the frequency of LANA:Blac+ (i.e., cleaved CCF2+ cells) that are B cells, DCs, SPM/monocytes, or LPMs at various times post infection. Each dot is representative of one mouse, and lines represent mean of samples. Data representative of three experiments with 2-3 mice per condition per experiment.

\section{The MHV68-Infected Peritoneal Cavity Contains Both Lytic and Latent Infection Early After Infection}

Our analysis of the cellular distribution of LANA:ßlac identified peritoneal macrophages as a prominent infected cell type at early time points post-infection. However, the state of virus infection in these cells remained in question. Though MHV68 has been reported to latently infect macrophages at late times post-infection (Weck et al., 1999), MHV68 has also been reported to lytically replicate in macrophages in vitro and in vivo (Tarakanova et al., 2007; Lawler et al., 2015). Additionally, it remained possible that the detection of LANA:ßlac in peritoneal macrophages may result from phagocytosis of other lytically infected cells rather than bona fide infection. To address this issue, we quantified the frequency of cells capable of producing virus using a limiting dilution-based assay, comparing virus production from intact and mechanically disrupted PerCs (Weck et al., 1996). In this assay, virus production from intact cells can result from either preformed, cell-associated virions or reactivation from latency. In contrast, mechanical disruption of cells prevents reactivation from latency, a process that requires viable cells, while having minimal impact on the detection of preformed virus (Weck et al., 1996). PerCs from mock, MHV68, 
A
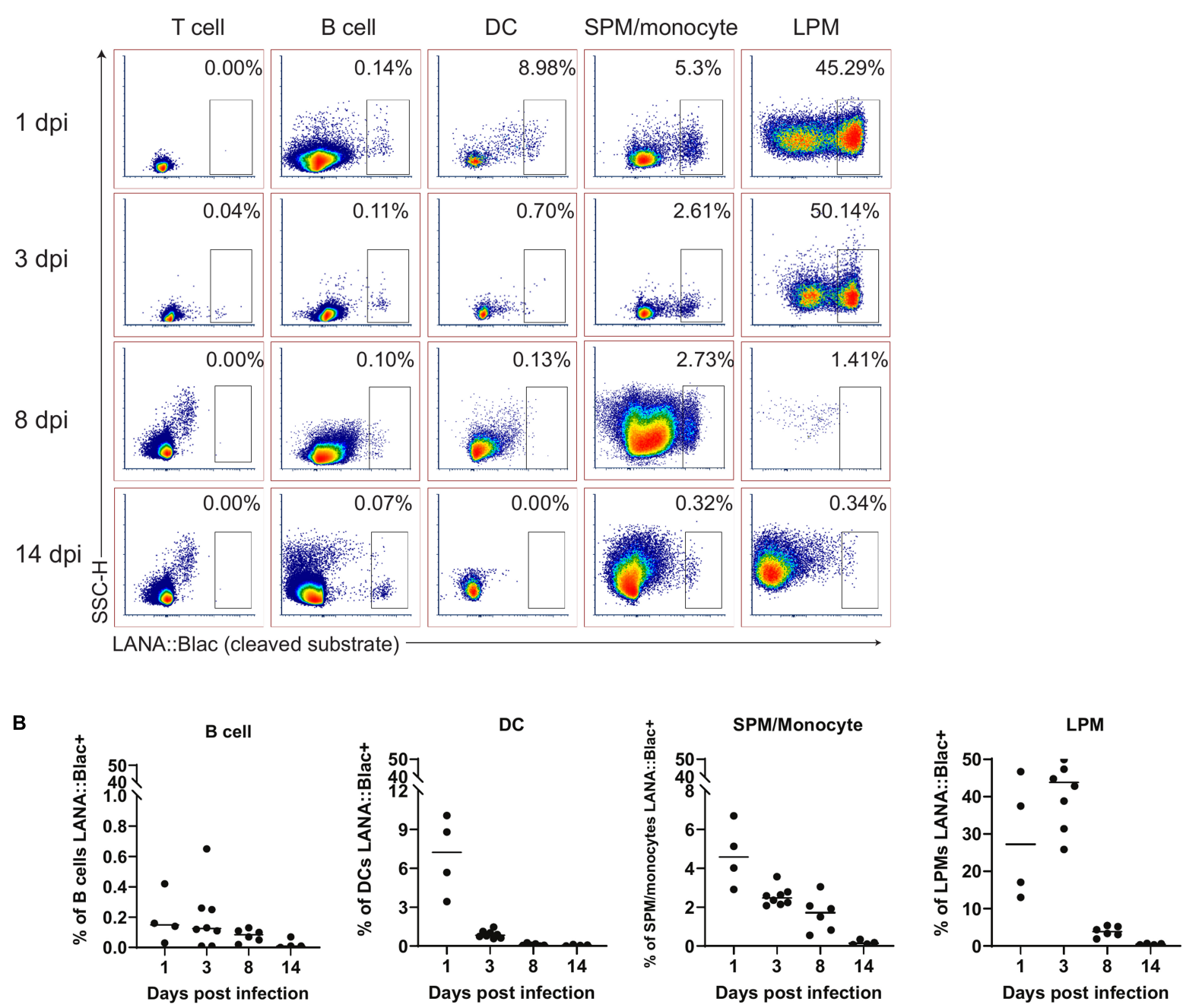

FIGURE 6 | A substantial portion of peritoneal macrophages are positive for MHV68.LANA: $\beta$ lac infection during acute infection. Flow cytometric analysis of cell subsets that demonstrate MHV68.LANA: $\beta$ lac infection in the peritoneal cavity of MHV68.LANA: $\beta$ lac infected mice. (A) Dot plots showing the frequency of T cells, B cells, dendritic cells, SPM/monocytes, and LPM that express cleaved CCF2 fluorescence, the indicator for LANA: $\beta$ lac expression, at various times post infection. (B) Graphs demonstrating data shown in (A). Each dot is representative of one mouse and lines represent mean of samples. Data representative of three experiments with 2-3 mice per condition per experiment.

or MHV68.LANA:ßlac-infected mice were isolated at $3 \mathrm{dpi}$ and plated in a series of dilutions on a MEF monolayer, a sensitive and permissive indicator for virus infection. Cocultures were observed for virus-induced CPE on MEF monolayers, quantifying preformed virus in mechanically disrupted PerCs and the sum of preformed virus and reactivation from latency in intact PerC co-cultures.

Peritoneal cavities isolated from MHV68- or MHV68.LANA:ßlac infected mice demonstrated virus-induced CPE in both intact and mechanically disrupted cells (Figure 8). In contrast, mock-infected PerCs demonstrated no detectable $\mathrm{CPE}$ in either condition (Figure 8). When we quantified the frequency of intact cells capable of producing virus, we found that $\sim 1$ in 9 PerCs $(\sim 11,95 \%$ confidence interval: $8.4-14 \%)$ were associated with virus production in both MHV68 and MHV-68.LANA:ßlac-infected mice. In contrast, limiting dilution analysis of mechanically disrupted cells identified a frequency of $\sim 1$ in 100 (1\%) of cells containing preformed virus (Figure 8). These data indicate that PerCs from MHV68-infected mice contain a mixture of latent and preformed virus, with latent infection comprising the dominant fraction of infected cells. Of note, the quantification of virus-infected cells determined via this functional assay was greater than that observed by flow cytometry at the same time point post infection $(\sim 11$ versus $\sim 5 \pm 0.6 \%$ ). Thus, consistent with previous reports (Nealy et al., 2010) assessment of infection frequency by LANA:ßlac cleavage and CCF2 fluorescence is likely an underestimate of total primary infection frequency. 


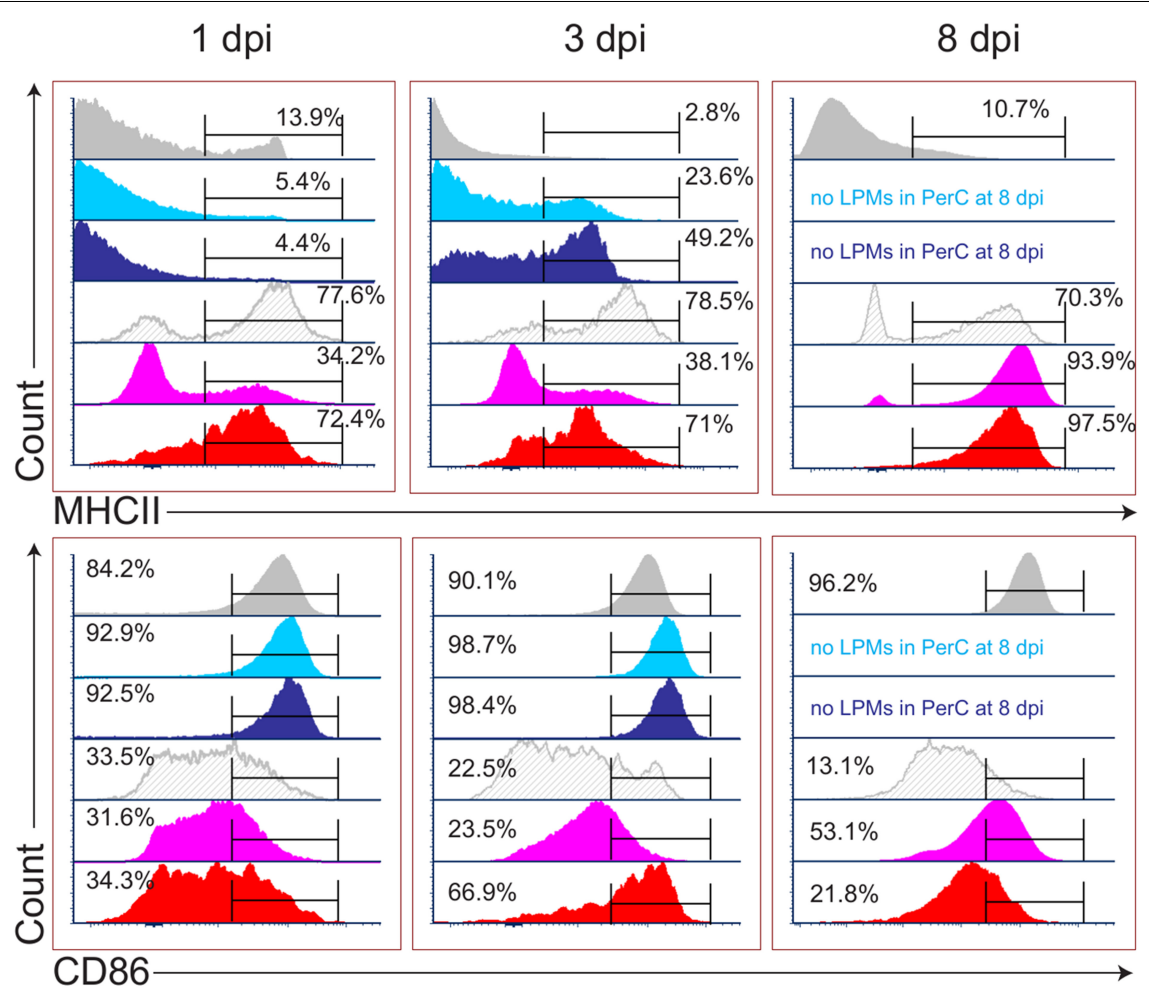

Mock LPM

LANA::Blac- LPM

LANA::Blac+ LPM

Mock SPM/monocyle

LANA::Blac- SPM/monocyte

LANA::Blac+ SPM/monocyte

FIGURE 7 | MHV68 infection is associated with altered macrophage expression of MHC II and CD86 in infected and uninfected cells. Flow cytometric analysis of MHC II (top row) and CD86 (bottom row) expression in large (LPM) and small (SPM) peritoneal macrophages, in mock or MHV68. LANA:Blac infected PerCs. Histograms compare the phenotype of virus-infected (LANA:ßlac+) and uninfected (LANA:ßlac-) cells. Gates define the percent of events positive for each marker. Virus-infected (LANA: $\beta l a c+)$ and uninfected (LANA:Blac-) cells were defined according to the gates drawn in Figure 6A. Data representative of three experiments with 2-3 mice per condition per experiment.

A

MHV68.LANA::Blac 3dpi

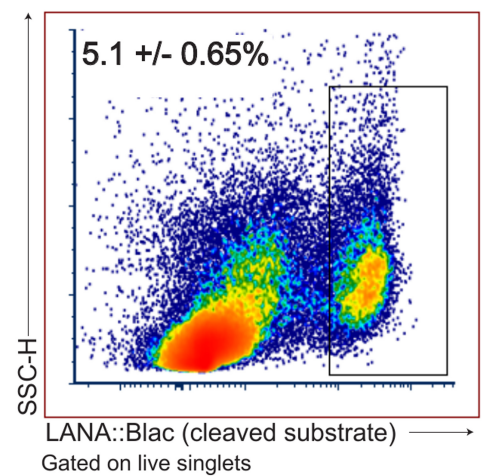

B

PerC 3 dpi

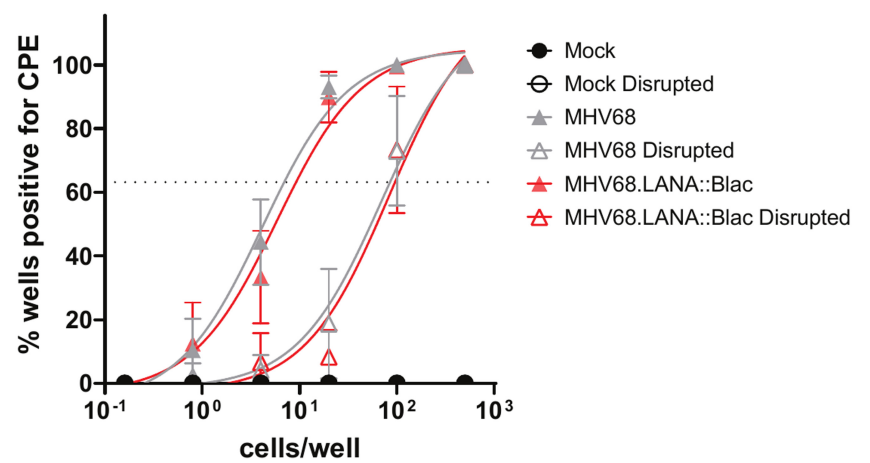

FIGURE 8 | The MHV68-infected peritoneal cavity contains both lytic and latent infection at 3 days post infection. (A) Flow cytometry analysis of peritoneal cells infected with MHV68.LANA:Blac. Shown is a representative dot plot of PerCs analyzed for cleaved CCF2 fluorescence at 3 dpi, gated on live singlets. Average frequency and standard error of the mean (upper left) were calculated from three experiments with 2-3 mice per condition per experiment. (B) Analysis of virus infection in peritoneal cells obtained from mock, MHV68 or MHV68.LANA:Blac infected mice. PerCs were harvested at 3 dpi and subjected to limiting dilution analysis on mouse embryonic fibroblasts, with virus production quantified by cytopathic effect (CPE) 21 days post-plating. Analysis quantifies the mean frequency of wells which were positive for virus in intact and mechanically disrupted samples. 24-36 wells per dilution per condition were analyzed for undisrupted samples, and 12 wells per dilution per condition for disrupted samples. Dashed line at $63.2 \%$ is used to determine the frequency of virus-positive cells, defined by Poisson distribution. Analysis performed via non-linear regression analysis in GraphPad Prism. Data representative of three experiments with two mice per condition per experiment. 


\section{DISCUSSION}

MHV68 is an established and efficient model virus for studying both pathogen and host elements of gammaherpesvirus infection. Though much progress has been made in characterizing the virus and the response it induces, its range of target cells, its dynamic viral lifecycle, and its low frequency of infected cells during latency make it difficult to track infection at the single-cell level. The MHV68.LANA:ßlac virus overcomes many of these issues by providing a highly sensitive, efficient, and robust readout of infected cells detected by flow cytometric analysis, allowing the rapid analysis of thousands of cells.

The MHV68.LANA:ßlac system has been previously used to identify infected cells (Coleman et al., 2010; Nealy et al., 2010; Diebel et al., 2015; Niemeyer et al., 2018) using conventional bandpass flow cytometry. In our study, we utilized full spectrum, spectral flow cytometry, a technology that bypasses restrictions of overlapping fluorescence emission spectra through the detection of unique emission profiles. We optimized CCF2-AM substrate and incubation conditions, allowing for detection of cleaved CCF2 fluorescence. Importantly, it is of note that virtually all cells positive for cleaved CCF2 fluorescence also retain an uncleaved CCF2 fluorescence spectrum, suggesting that betalactamase expressing cells are unable to cleave every molecule of CCF2-AM within the cytoplasm. It is unclear whether there is an ideal concentration of CCF2-AM substrate that would allow for complete cleavage by beta-lactamase, and whether this would be desirable, given that detection of the uncleaved substrate also provides valuable evidence of equal substrate loading. Following identification of these optimized conditions, we were able to measure CCF2-AM fluorescence in concert with a panel of twelve surface markers, a major advance in utility of this reporter system.

With the optimization of MHV68.LANA:Blac detection by spectral flow cytometry, we characterized the impact of acute MHV68 infection on cells in the PerC, examining both the broad impact of MHV68 infection as well as the specific targets of MHV68 infection. We found that MHV68 infection is associated with pronounced changes in the cellular composition of PerCs, with rapid increases in the frequency and number of CD8 $\mathrm{T}$ cells, and pronounced changes to myeloid cells, including transient elimination of LPMs coupled with expansion of SPMs and monocytes. MHV68 infection was further associated with long-term phenotypic changes in LPMs and SPMs, including upregulation of MHC II expression in LPMs.

One of the most pronounced changes in the PerC was the transient depletion of LPMs. The disappearance of LPMs, a phenomenon referred to as the "macrophage disappearance reaction," is a well-known outcome in response to inflammatory stimuli, including thioglycollate, LPS, virus, and infection with the parasite Trypanosoma cruzi (Ghosn et al., 2010; Cassado Ados et al., 2015; Gautam et al., 2019). During this response, LPMs are presumed to traffic to the omentum, where they potentially carry antigen to stimulate B cells to mature in milky spots, small lymphoid structures within the omentum (Rangel-Moreno et al., 2009). As the omentum is a highly vascularized tissue, it is also possible that macrophages further disseminate to the lymphatics or the bloodstream (Meza-Perez and Randall, 2017).
We did not see any sign of death in LPMs before elimination, nor did we observe their presence when adding additional EDTA to our extraction solution (data not shown), suggesting that the loss of LPMs is not due to their lysis or adherence to the peritoneal walls.

Despite the depletion of LPMs at $8 \mathrm{dpi}$, LPM-like cells reappeared in the infected PerC by $14 \mathrm{dpi}$. It is unclear how this subset is repopulated, as LPMs were originally described as tissue-resident and self-renewing (Ghosn et al., 2010). One alternate explanation could be that LPMs are replaced by SPMs or monocytes, as these cell subsets exhibit similar F4/80 and CD11b expression during the recovery phase, with LPM and SPM characterized by relatively comparable expression of MHC II and CD86 at later times post-infection. The potential replacement of LPMs by SPMs or monocytes is supported by previous studies (Cain et al., 2013; Yona et al., 2013; Bain et al., 2016) and represents an important unresolved question in the context of MHV68 infection. Delineation between these two populations during infection could be furthered clarified by staining for other discriminating markers, such as ICAM-2 or TIM-4.

In addition to observing widespread changes in peritoneal cell composition, our studies allowed detailed analysis of targets of early MHV68 infection in the PerC. We observed a robust level of infection, with as many as $8 \%$ of peritoneal cells infected at 1 day post infection. We note that these numbers are likely an underestimate of the total number of virally infected cells, as not all virally infected cells will express LANA at a given time point (Nealy et al., 2010; Niemeyer et al., 2018), and there are likely cells that are unable to produce functional LANA:ßlac enzyme. Furthermore, some virally infected cells may be too fragile to survive the lengthy staining protocol and harsh fluidics of flow cytometry. Indeed, our limiting dilution analyses at 3dpi identified a greater percentage of infected cells $(\sim 11 \%)$ than identified by flow cytometry $(\sim 5 \%)$.

Our analysis revealed a high frequency of infection (25-40\%) in LPMs through 3 dpi. At 8 dpi, the SPM/monocyte population becomes the predominant source of infection. We note that the majority of LANA:Blac+ within the SPM/monocyte population express high levels of MHCII, suggesting these cells are SPMs rather than monocytes (Ghosn et al., 2010). Dendritic cells and $B$ cells represented a minor fraction of LANA:ßlac + events. The presence of virus in antigen presenting cells during early infection in the PerC is an exciting finding, as these cells have the ability to initiate local antigen-specific immune responses within the cavity as well as the potential to disseminate virus to other tissues via trafficking to lymphoid tissues.

We further identified that virus infection resulted in changes to macrophage phenotype, both within virus-infected cells and in uninfected cells. Both LANA:ßlac+ and LANA:ßlacmacrophages displayed upregulation of the activation markers MHC II and CD86, a trend that was most prevalent within LANA:Blac+ populations. It is unclear whether viral infection of these cells induces upregulation of activation markers, or alternatively, whether activated macrophages are more likely viral targets. Regardless, upregulation of these markers has functional consequences for peritoneal macrophages. High expression of MHC II expression suggests that these cells may either be 
activated and/or directly capable of presenting antigen to virusspecific CD4 T cells.

The observation that MHV68 is predominant in LPMs before their disappearance at $8 \mathrm{dpi}$ also suggests that MHV68 may potentially exploit LPM responses to inflammation to facilitate virus dissemination to the omentum. Consistent with this, previous studies by Gray et al. (2012) identified MHV68+ cells in the omentum, including B220 + B cells and a smaller fraction of CD11b+ cells, presumed to be macrophages. It is also possible that MHV68+ LPMs either undergo lytic replication and/or are directly targeted for destruction by antiviral T cells.

It is notable that early infection contained a mixture of latently infected cells and cells undergoing lytic replication, with the vast majority of infected peritoneal cells containing latent virus. The detection of latency is often precluded by the presence of lytic infection, however high frequencies of latency at an early time point of 3 dpi suggests direct latent infection of target cells, in this case macrophages. This early establishment of latency is consistent with a previous report identifying the early establishment of latency in the lung following intranasal infection (Flano et al., 2005). While this analysis focused on bulk PerCs, future studies, purifying LANA:ßlac+ peritoneal macrophages may provide further evidence for latent infection during these early stages of infection and may help to understand host cues that initiate viral latency.

We note that at $14 \mathrm{dpi}$, the LANA: $\beta$ lac is not well detected above background. Previous studies using this reporter virus reported LANA:ßlac+ cells at less than or equal to $0.1 \%$ of splenocytes at 16 dpi (Nealy et al., 2010; Lange et al., 2019), and limiting dilution qPCR experiments have reported frequencies of less than $1 \%$ infected cells in specific cell populations in the PerC at 16 dpi (Rekow et al., 2016). Based on these data, we infer LANA: $\beta$ lac + cells are a rare but present population within the PerC at 14 days or later post infection. These cells may be more effectively detected by greatly increasing the number of peritoneal cells analyzed and/or by enriching for cell types known to be infected prior to analysis.

In total, this study demonstrates optimization of the MHV68.LANA:ßlac reporter system, to identify targets of virus infection using spectral flow cytometry. By tracking MHV68 infection in the PerC, this analysis identified both broad changes in the immune landscape of the PerC as well as differential protein expression profiles in virus-infected and uninfected cells. These studies identify MHV68-induced myeloid reprogramming, characterized by the induction of MHC II in LPMs and CD86 induction in SPMs, consistent with virus-induced alterations to the PerC previously associated with MHV68-induced crossprotection against bacterial infection (Barton et al., 2007). We further identify peritoneal macrophages as a prominent target of acute infection and identify a mixture of lytic and latent infection early post-infection, with a striking dominance of latent infection. This work offers an optimized technique for high throughput single-cell immunophenotyping of virally infected cells, emphasizes the dynamic interactions between MHV68 and the host, and provide critical cellular context that will allow future analysis of factors that regulate early virus-host dynamics.

\section{DATA AVAILABILITY STATEMENT}

The original contributions presented in the study are included in the article/Supplementary Material, further inquiries can be directed to the corresponding author/s.

\section{ETHICS STATEMENT}

The animal study was reviewed and approved by the Institutional Animal Care and Use Committee.

\section{AUTHOR CONTRIBUTIONS}

JR, EC, LD, and LB designed and conceived this project and wrote the manuscript. JR performed the experiments, analyzed the data, and prepared the figures. EM, LB, and DB provided insight in to experimental design and data analysis. All authors read and approved the final manuscript.

\section{FUNDING}

Funding for this work was provided by NIH grant AI32419 (LB). The Flow Cytometry Shared Resources of the University of Colorado Cancer Center receive direct funding support from the National Cancer Institute through Cancer Center Support Grant: P30CA046934.

\section{ACKNOWLEDGMENTS}

We thank the University of Colorado Anschutz School of Medicine Office of Laboratory Animal Resources (OLAR) and Alexander Sosa for technical support with animal husbandry. We would also like to thank the University of Colorado Cancer Center Flow Cytometry Shared Resource as well as the Department of Immunology and Microbiology Flow Cytometry Shared Resource Lab for assistance with flow cytometry.

\section{SUPPLEMENTARY MATERIAL}

The Supplementary Material for this article can be found online at: https://www.frontiersin.org/articles/10.3389/fmicb.2021. 656979/full\#supplementary-material

Supplementary Figure 1 | MHV68.LANA: $\beta$ lac is detectable in B cells at 8 days post infection in the spleen. C57BL/6 mice were infected i.p. with 1 million PFUs of WT MHV68 or MHV68.LANA: $\beta$ lac. At 1, 3, 8, and $14 \mathrm{dpi}$, mice were sacrificed and spleens were harvested, counted, and stained with CCF2-AM, viability, and surface markers. (A) Viable cell counts of harvested spleens in mock, MHV68 or MHV68.LANA: $\beta$ lac infected mice. (B) Dot plots showing the frequency of splenocytes positive for cleaved CCF2. LANA: $\beta$ lac signal is enriched above background (defined by WT MHV68 infection) at 8 dpi. (C) Quantification of the mean frequency and number of LANA: $\beta$ lac+ cells shown in (B). (D) Dot plots showing percentage of T cells, B cells, dendritic cells, and macrophages that are positive for cleaved CCF2. B cells are the only population with cleaved CCF2 signal, with about $0.5 \%$ of cells staining positive. Data representative of three experiments with 2-3 mice per condition per experiment. 
Supplementary Figure 2 | Gating strategy for analysis of spectral flow cytometry data. Representative gating scheme adopted from Ghosn et al. (2010). Sample

\section{REFERENCES}

Bain, C. C., Hawley, C. A., Garner, H., Scott, C. L., Schridde, A., Steers, N. J., et al. (2016). Long-lived self-renewing bone marrow-derived macrophages displace embryo-derived cells to inhabit adult serous cavities. Nat. Commun. 7:ncomms11852. doi: 10.1038/ncomms11852

Barton, E., Mandal, P., and Speck, S. H. (2011). Pathogenesis and host control of gammaherpesviruses: lessons from the mouse. Annu. Rev. Immunol. 29, 351-397. doi: 10.1146/annurev-immunol-072710-081639

Barton, E. S., White, D. W., Cathelyn, J. S., Brett-McClellan, K. A., Engle, M., Diamond, M. S., et al. (2007). Herpesvirus latency confers symbiotic protection from bacterial infection. Nature 447, 326-329. doi: 10.1038/nature05762

Baumgarth, N. (2017). A Hard(y) Look at B-1 Cell Development and Function. J. Immunol. 199, 3387-3394. doi: 10.4049/jimmunol.1700943

Berger, J. N., Sanford, B., Kimball, A. K., Oko, L. M., Kaspar, R. E., Niemeyer, B. F., et al. (2020). Redefining de novo gammaherpesvirus infection through high-dimensional, single-cell analysis of virus and host. bioRxiv [Preprint]. doi: $10.1101 / 2020.08 .11 .203117$

Cain, D. W., O’Koren, E. G., Kan, M. J., Womble, M., Sempowski, G. D., Hopper, K., et al. (2013). Identification of a tissue-specific, C/EBPbeta-dependent pathway of differentiation for murine peritoneal macrophages. J. Immunol. 191, 4665-4675. doi: 10.4049/jimmunol.1300581

Cassado Ados, A., D’Imperio Lima, M. R., and Bortoluci, K. R. (2015). Revisiting mouse peritoneal macrophages: heterogeneity, development, and function. Front. Immunol. 6:225. doi: 10.3389/fimmu.2015.00225

Cesarman, E., Damania, B., Krown, S. E., Martin, J., Bower, M., and Whitby, D. (2019). Kaposi sarcoma. Nat. Rev. Dis. Primers 5:9. doi: 10.1038/s41572-0190060-9

Cho, H. S., Ha, S., Shin, H. M., Reboldi, A., Hall, J. A., Huh, J. R., et al. (2020). CD8(+) T Cells Require ITK-Mediated TCR Signaling for Migration to the Intestine. Immunohorizons 4, 57-71. doi: 10.4049/immunohorizons. 1900093

Cho, H. S., Reboldi, A., Hall, J. A., and Berg, L. J. (2019). The Tec kinase ITK is essential for ILC2 survival and epithelial integrity in the intestine. Nat. Commun. 10:784. doi: 10.1038/s41467-019-08699-9

Coleman, C. B., Nealy, M. S., and Tibbetts, S. A. (2010). Immature and transitional B cells are latency reservoirs for a gammaherpesvirus. J. Virol. 84, 13045-13052. doi: 10.1128/JVI.01455-10

Collins, C. M., Boss, J. M., and Speck, S. H. (2009). Identification of infected B-cell populations by using a recombinant murine gammaherpesvirus 68 expressing a fluorescent protein. J. Virol. 83, 6484-6493. doi: 10.1128/JVI.00297-09

Collins, C. M., Scharer, C. D., Murphy, T. J., Boss, J. M., and Speck, S. H. (2020). Murine gammaherpesvirus infection is skewed toward Iglambda+ B cells expressing a specific heavy chain V-segment. PLoS Pathog. 16:e1008438. doi: 10.1371/journal.ppat.1008438

Collins, C. M., and Speck, S. H. (2012). Tracking murine gammaherpesvirus 68 infection of germinal center B cells in vivo. PLoS One 7:e33230. doi: 10.1371/ journal.pone.0033230

Diebel, K. W., Oko, L. M., Medina, E. M., Niemeyer, B. F., Warren, C. J., Claypool, D. J., et al. (2015). Gammaherpesvirus small noncoding RNAs are bifunctional elements that regulate infection and contribute to virulence in vivo. mBio 6:e01670-14. doi: 10.1128/mBio.01670-14

Dittmer, D. P., and Damania, B. (2016). Kaposi sarcoma-associated herpesvirus: immunobiology, oncogenesis, and therapy. J. Clin. Invest. 126, 3165-3175. doi: 10.1172/JCI84418

Farrell, P. J. (2019). Epstein-Barr virus and cancer. Annu. Rev. Pathol. 14, 29-53. doi: 10.1146/annurev-pathmechdis-012418-013023

Flano, E., Husain, S. M., Sample, J. T., Woodland, D. L., and Blackman, M. A. (2000). Latent murine gamma-herpesvirus infection is established in activated B cells, dendritic cells, and macrophages. J. Immunol. 165, 1074-1081. doi: 10.4049/jimmunol.165.2.1074

Flano, E., Jia, Q., Moore, J., Woodland, D. L., Sun, R., and Blackman, M. A. (2005). Early establishment of gamma-herpesvirus latency: implications for immune control. J. Immunol. 174, 4972-4978. doi: 10.4049/jimmunol.174.8.4972 derived from PerCs of a mock-infected mouse. FSC histogram denotes size difference between SPM/monocyte and LPM populations.
Frederico, B., Milho, R., May, J. S., Gillet, L., and Stevenson, P. G. (2012). Myeloid infection links epithelial and B cell tropisms of Murid Herpesvirus-4. PLoS Pathog. 8:e1002935. doi: 10.1371/journal.ppat.1002935

Gautam, A., Park, B. K., Kim, T. H., Akauliya, M., Kim, D., Maharjan, S., et al. (2019). Peritoneal cells mediate immune responses and cross-protection against influenza A virus. Front. Immunol. 10:1160. doi: 10.3389/fimmu.2019.01160

Ghosn, E. E., Cassado, A. A., Govoni, G. R., Fukuhara, T., Yang, Y., Monack, D. M., et al. (2010). Two physically, functionally, and developmentally distinct peritoneal macrophage subsets. Proc. Natl. Acad. Sci. U.S.A. 107, 2568-2573. doi: 10.1073/pnas.0915000107

Gray, K. S., Collins, C. M., and Speck, S. H. (2012). Characterization of omental immune aggregates during establishment of a latent gammaherpesvirus infection. PLoS One 7:e43196. doi: 10.1371/journal.pone.0043196

Hwang, S., Wu, T. T., Tong, L. M., Kim, K. S., Martinez-Guzman, D., Colantonio, A. D., et al. (2008). Persistent gammaherpesvirus replication and dynamic interaction with the host in vivo. J. Virol. 82, 12498-12509. doi: 10.1128/JVI. 01152-08

Knapp, T., Hare, E., Feng, L., Zlokarnik, G., and Negulescu, P. (2003). Detection of beta-lactamase reporter gene expression by flow cytometry. Cytometry A 51, 68-78. doi: $10.1002 /$ cyto.a.10018

Lange, P. T., Jondle, C. N., Darrah, E. J., Johnson, K. E., and Tarakanova, V. L. (2019). LXR alpha restricts gammaherpesvirus reactivation from latently infected peritoneal cells. J. Virol. 93:e02071-18. doi: 10.1128/JVI.020 71-18

Lawler, C., Milho, R., May, J. S., and Stevenson, P. G. (2015). Rhadinovirus host entry by co-operative infection. PLoS Pathog. 11:e1004761. doi: 10.1371/journal. ppat.1004761

Meza-Perez, S., and Randall, T. D. (2017). Immunological functions of the omentum. Trends Immunol. 38, 526-536. doi: 10.1016/j.it.2017. 03.002

Nealy, M. S., Coleman, C. B., Li, H., and Tibbetts, S. A. (2010). Use of a virus-encoded enzymatic marker reveals that a stable fraction of memory B cells expresses latency-associated nuclear antigen throughout chronic gammaherpesvirus infection. J. Virol. 84, 7523-7534. doi: 10.1128/JVI.025 72-09

Niemeyer, B. F., Oko, L. M., Medina, E. M., Oldenburg, D. G., White, D. W., Cool, C. D., et al. (2018). Host tumor suppressor p18(INK4c) functions as a potent cell-intrinsic inhibitor of murine gammaherpesvirus 68 reactivation and pathogenesis. J. Virol. 92, JVI.1604-JVI.1617. doi: 10.1128/JVI.01604-17

Nolan, J. P., and Condello, D. (2013). Spectral flow cytometry. Curr. Protoc. Cytom. 63, 1.27.1-1.27.13. doi: 10.1002/0471142956.cy0127s63

Nolan, J. P., Condello, D., Duggan, E., Naivar, M., and Novo, D. (2013). Visible and near infrared fluorescence spectral flow cytometry. Cytometry A 83, 253-264. doi: 10.1002/cyto.a.22241

Peacock, J. W., and Bost, K. L. (2000). Infection of intestinal epithelial cells and development of systemic disease following gastric instillation of murine gammaherpesvirus-68. J. Gen. Virol. 81(Pt. 2), 421-429. doi: 10.1099/00221317-81-2-421

Rangel-Moreno, J., Moyron-Quiroz, J. E., Carragher, D. M., Kusser, K., Hartson, L., Moquin, A., et al. (2009). Omental milky spots develop in the absence of lymphoid tissue-inducer cells and support $\mathrm{B}$ and $\mathrm{T}$ cell responses to peritoneal antigens. Immunity 30, 731-743. doi: 10.1016/j.immuni.2009.03.014

Ray, A., and Dittel, B. N. (2010). Isolation of mouse peritoneal cavity cells. J. Vis. Exp. 35:1488. doi: 10.3791/1488

Rekow, M. M., Darrah, E. J., Mboko, W. P., Lange, P. T., and Tarakanova, V. L. (2016). Gammaherpesvirus targets peritoneal B-1 B cells for long-term latency. Virology 492, 140-144. doi: 10.1016/j.virol.2016.02.022

Rosa, G. T., Gillet, L., Smith, C. M., de Lima, B. D., and Stevenson, P. G. (2007). IgG fc receptors provide an alternative infection route for murine gamma-herpesvirus-68. PLoS One 2:e560. doi: 10.1371/journal.pone.000 0560

Shannon-Lowe, C., and Rickinson, A. (2019). The global landscape of EBVassociated tumors. Front. Oncol. 9:713. doi: 10.3389/fonc.2019.00713 
Suarez, A. L., and van Dyk, L. F. (2008). Endothelial cells support persistent gammaherpesvirus 68 infection. PLoS Pathog. 4:e1000152. doi: 10.1371/journal. ppat. 1000152

Sunil-Chandra, N. P., Efstathiou, S., Arno, J., and Nash, A. A. (1992). Virological and pathological features of mice infected with murine gamma-herpesvirus 68 . J. Gen. Virol. 73(Pt. 9), 2347-2356. doi: 10.1099/0022-1317-73-9-2347

Tarakanova, V. L., Leung-Pineda, V., Hwang, S., Yang, C. W., Matatall, K., Basson, M., et al. (2007). Gamma-herpesvirus kinase actively initiates a DNA damage response by inducing phosphorylation of $\mathrm{H} 2 \mathrm{AX}$ to foster viral replication. Cell Host Microbe 1, 275-286. doi: 10.1016/j.chom.2007.05.008

Weck, K. E., Barkon, M. L., Yoo, L. I., Speck, S. H., and Virgin, H. I. (1996). Mature $B$ cells are required for acute splenic infection, but not for establishment of latency, by murine gammaherpesvirus 68. J. Virol. 70, 6775-6780. doi: 10.1128/ JVI.70.10.6775-6780.1996

Weck, K. E., Kim, S. S., Virgin, H. I., and Speck, S. H. (1999). Macrophages are the major reservoir of latent murine gammaherpesvirus 68 in peritoneal cells. J. Virol. 73, 3273-3283. doi: 10.1128/JVI.73.4.3273-3283.1999

Yona, S., Kim, K. W., Wolf, Y., Mildner, A., Varol, D., Breker, M., et al. (2013). Fate mapping reveals origins and dynamics of monocytes and tissue macrophages under homeostasis. Immunity 38, 79-91. doi: 10.1016/j.immuni.2012. 12.001
Young, L. S., Yap, L. F., and Murray, P. G. (2016). Epstein-Barr virus: more than 50 years old and still providing surprises. Nat. Rev. Cancer 16, 789-802. doi: $10.1038 /$ nrc. 2016.92

Zlokarnik, G., Negulescu, P. A., Knapp, T. E., Mere, L., Burres, N., Feng, L., et al. (1998). Quantitation of transcription and clonal selection of single living cells with beta-lactamase as reporter. Science 279, 84-88. doi: 10.1126/science.279. 5347.84

Conflict of Interest: DB was employed by company Cytek Biosciences.

The remaining authors declare that the research was conducted in the absence of any commercial or financial relationships that could be construed as a potential conflict of interest.

Copyright (C) 2021 Riggs, Medina, Perrenoud, Bonilla, Clambey, van Dyk and Berg. This is an open-access article distributed under the terms of the Creative Commons Attribution License (CC BY). The use, distribution or reproduction in other forums is permitted, provided the original author(s) and the copyright owner(s) are credited and that the original publication in this journal is cited, in accordance with accepted academic practice. No use, distribution or reproduction is permitted which does not comply with these terms. 